\title{
Design of MPPT controller based on battery fast charging method in photovoltaic system
}

\author{
Ding Chen ${ }^{1, a}$, Yubao Gao ${ }^{1, b,{ }^{*}}$ \\ ${ }^{1}$ School of Electrical Engineering, Jiujiang University, Jiujiang, 332005, China \\ aemail: chending11love@163.com, bemail:ybgao_ju@163.com \\ ${ }^{*}$ corresponding author
}

Keywords: MPPT; Fast charging; Photovoltaic system

\begin{abstract}
MPPT algorithms have been widely used in controllers of photovoltaic system. While for independent photovoltaic systems, researches focused on the charging method to batteries are still limited. Considering the power supply in continual rainy days, the capacity redundancy o batteries in photovoltaic system should be considered. However, it is difficult to fully charge these batteries at one time, which results that these batteries usually work in undervoltage conditions and then will be damaged in performance. Based on the characteristics of the batteries charging and discharging, a controller design combining battery fast charging and MPPT algorithm is proposed in this paper. Using the controller, not only MPPT function, but also protection on battery can be realized in photovoltaic systems. Furthermore, due to decrease of batteries maintenance cost, the comprehensive utilization efficiency of energy can be improved.
\end{abstract}

\section{Introduction}

Independent photovoltaic (hereafter referred as PV) systems, such as solar street lamp, solar insect-killing lamp and so on, have a wide application prospect in areas far from the power grid. This type of power system must be equipped with battery to storage the solar energy in daytime, for working in nights. To design such systems, capacity redundant of battery must be considered to maintain working during continual rainy days. In this situation, to make full use of solar energy, various Maximum Power Point Tracking (hereafter referred as MPPT) algorithms have been developed based on the characteristics of solar cells output. Xiao et al. [1] have studied on Perturbation and Observation (hereafter referred as $\mathrm{P} \& \mathrm{O}$ ) and climbing methods for reducing the oscillation near the PV modules Maximum Power Point (hereafter referred as MPP). And then a variable perturbation step has been proposed in their researches. When the operating point is close to the MPP, the step gets smaller. Hsiao et al. [2] have worked on the failure of P\&O algorithm in the situation of rapidly changing atmosphere and then proposed a three-point weight comparison method used in $\mathrm{P} \& \mathrm{O}$ algorithm. Hussain et al. [3], by comparing the instantaneous and incremental conductance of PV modules, proposed a MPPT algorithm to accurately track the MPP of solar cells even in the situation of rapidly changing atmosphere. Patcharaprakiti et al. [4] used an adaptive fuzzy logic method to optimize the performance of MPPT algorithm. Parimita et al. [5] compared the performances of several existing MPPT methods in a solar system in view of conversion efficiency, energy captured, reliability etc. In these literatures, researchers focused on the performance of PV modules output, while ignored the protection of battery.

In independent PV system, widely used energy storage module is Valve-Regulated Lead Acid (hereafter referred as VRLA) battery which generally works about 3 years. VRLA batteries have specific charging and discharging characteristics. If the charging process deviate the characteristics, the service time of batteries will be shorten obviously. For both the productive process and the scrap processing of VRLA batteries will produce environmental pollution, it is costly for maintaining the PV system by renewing VRLA batteries frequently. Researches indicated that the charging and discharging processes significantly affect the service period of VRLA batteries, especially the charging process [6]. Therefore, researchers developed multi-stage charging methods based on MPPT algorithms in terms of the battery charging characteristics. For instance, Xiao et al. [7] 
studied two-stage charging method for VRLA batteries in PV systems. Wang et al. [8] presented three-stage battery charging technology used in wind and solar control systems. However, these methods charge batteries slowly, which can't meet the charging requirement for batteries capacity redundant to maintain working during continual rainy days. Thus it is difficult to charge the batteries fully at one time by using the MPPT technology with common charging methods. For this reason, the batteries usually work in undervoltage condition, which will significantly shorten its service time. This paper proposes a design method for MPPT controller adopted a fast charging strategy in terms of the batteries charging and discharging characteristics. By the means provided in this paper, the solar energy maximizing use and the batteries protection can be achieve a balance, and then the entire efficiency of energy utilization can be improved.

\section{MPPT algorithm}

A PV system with MPPT function is shown as Fig. 1.

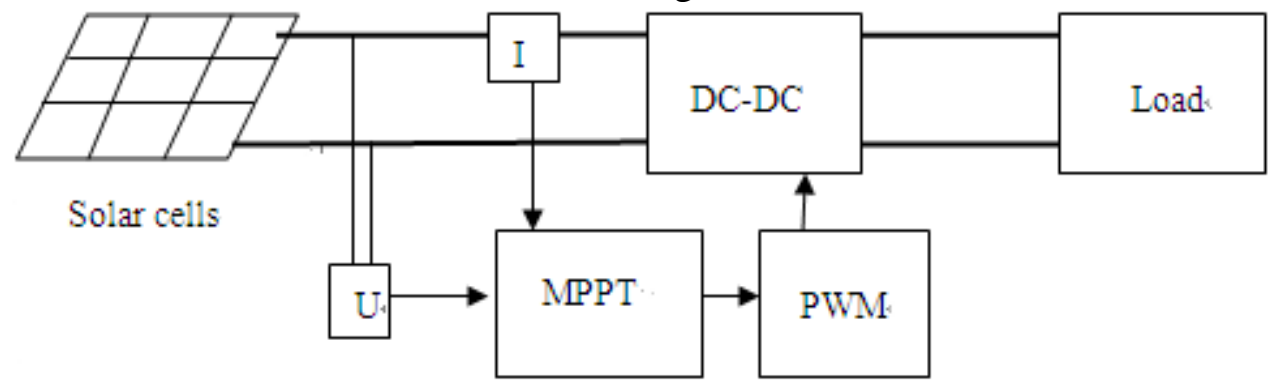

Fig.1. Diagram of a PV system with MPPT function

The relationship curve between the power and the voltage outputs of solar cells is shown as Fig. 2.

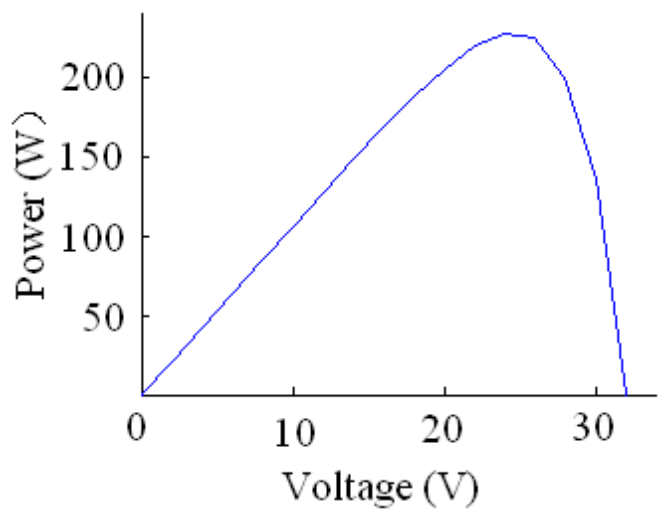

Fig. 2 Solar cells P-V curve

The peak of curve in Fig.2 is the MPP of solar cells. Obviously, the MPP isn't the maximum voltage of solar cells. To realize that the PV modules always work at the MPP, various MPPT algorithms have been developed. Among these algorithms, Perturbation and Observation (P\&O) algorithm is widely used for simplicity and easy realization. The principle of $\mathrm{P} \& \mathrm{O}$ algorithm is described as following.

Let $I$ represent the output current of solar cells, and $U$ represent the voltage, then the power can be obtained by

$P=U I$

In view of Fig. 2, when

$\mathrm{d} P / d U=0$

i.e. at the peak of curve in Fig. 2, the solar cells work at the MPP. When

$\mathrm{d} P / d U>0$

the $U$ should be increased to let the work point close to the MPP. Similarly, when

$\mathrm{d} P / d U<0$

the $U$ should be decreased to make the trend of work point changing close to the MPP. 
There are two situations in Eq. (3): both $U$ and $P$ are increasing, or $U$ and $P$ are all decreasing. Similarly, two situations lie in Eq. (4): $U$ is increasing when $P$ decreasing, or $U$ is decreasing when $P$ increasing.

The $U$ can be changed by Pulse-Width Modulation (PWM, shown in Fig. 1) signal, which regulates the impedance of circuit. By changing the duty cycle of PWM signal, the MPPT algorithm changes the $U$, and then measures the $I$ shown in Fig. 2. In terms of Eqs. (1)-(4), for tracking the MPP of solar cells, the changing trend of $U$ can be obtained.

\section{Charging and discharging characteristics of VRLA batteries}

The acceptable current of VRLA batteries follows below equation [9]

$$
I_{\mathrm{a}}=I_{\mathrm{s}} e^{-\alpha t}
$$

where $I_{s}$ is the maximum acceptable current at $\mathrm{t}=0$, and $\alpha$ is decay rate.

Let $I_{\mathrm{C}}$ represent the charging current. In charging process, if $I_{\mathrm{c}}=I_{\mathrm{a}}, I_{\mathrm{c}}$ will be fully transformed into energy storage by the battery, and no gas separates out to waste energy. If $I_{\mathrm{c}}>I_{\mathrm{a}}$, the extra current will be transformed into gas to separate out. While if $I_{\mathrm{c}}<I_{\mathrm{a}}$, the charging period will be prolonged. Thus the optimal charging current should be match equation (5).

In PV system, it is difficult to measure the charging current in time. So researchers proposed a multi-stage charging method. The threshold values for separating charging stages are the terminal voltage of the battery. Using this means, it is easy to realize that the charging current is no more than $I_{\mathrm{a}}$. While based on Eq. (5), it is obvious that $I_{\mathrm{a}}$ decay rapidly with exponent rule. This denotes that the charging time with large current lasts shortly. Therefore, the charging speed of multi-stage charging method based on Eq. (5) is slow, which usually can't satisfy the charging requirement for solar energy system. This limitation results that the batteries usually work in undervoltage state, and significantly reduces its service period.

\section{New MPPT approach based on fast charging model}

Based on literature [9], Mass derived the relation between acceptable charging current and discharging current of barriers, which is depicted as

$$
\alpha=K \cdot \log \left(k I_{d}\right)
$$

where $I_{d}$ represent discharging current, and $K$ is constant.

Eqs. (5) and (6) indicate that the larger the discharging current is, the stronger the acceptable capability of charging current is. It denotes that the $I_{d}$ has a absolute affection to the $I_{\mathrm{a}}$. Therefore, if the battery is discharged intermittently during its charging period, the capability of charging with large current can be strengthened. By this means, the lasting time with large charging current can be prolonged, and then the fast charging can be realized.

In summary, to eliminate the polarization phenomena during battery charging process, a set of pulse signals can be used intermittently to discharge the battery deeply with a very fast period [10]. Then the efficiency of battery charging can be improved. Using this principle in a PV system, the MPPT controller can be designed with such a strategy: (1) when the battery terminal voltage is lower than the threshold value of fast charging model, the controller adopts MPPT algorithm to charge the battery with the maximum power of solar cells. (2) when the battery terminal voltage arrived at the threshold value, the controller implements fast charging model in which the battery is deeply discharged intermittently with a very fast period during the charging process. 


\section{Design of the new MPPT controller}

The diagram of a PV system with the MPPT controller including battery fast charging model is shown as Fig. 3.

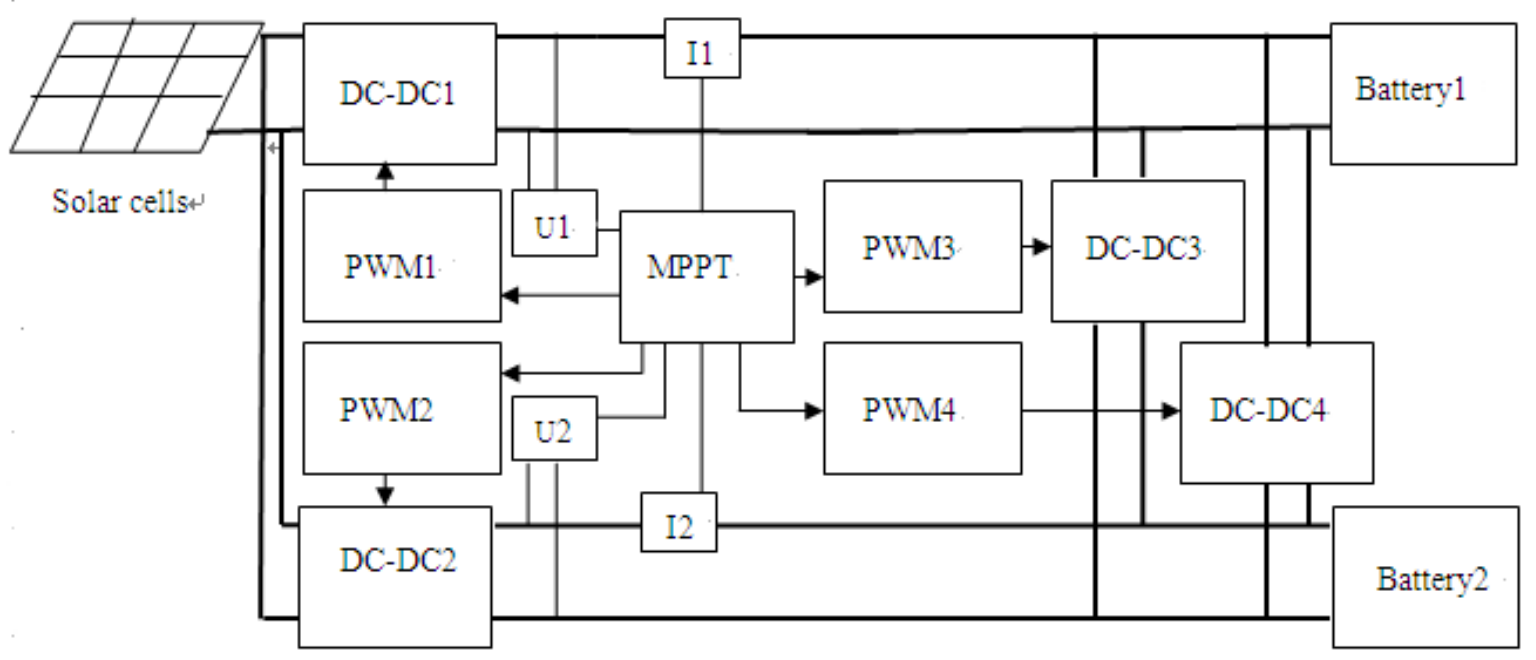

Fig.3 Diagram of a PV system with new MPPT controller

In Fig.3, the batteries are divided into two groups. The power of solar cells can be charged into group 1 and group 2 separately through DC-DC1 and DC-DC2 modules. When the batteries terminal voltages are under the threshold value of fast charging model, the MPPT controller drive DC-DC 1 and DC-DC 2 separately through PWM1 and PWM2 modules using a MPPT algorithm (e.g. P\&O algorithm), and stop DC-DC3 and DC-DC4 by PWM3 and PWM4 modules, respectively. In this situation, the solar cells can work at MPP. As shown in Fig.3, when using P\&O algorithm with such topology in a PV system, for the batteries terminal voltages are relatively steady, the perturbation variable is charging current. When group 1 of batteries achieved the threshold value of fast charging model, the controller adopts pulse charging model with such a work sequence: DC-DC1 is driven by PWM1 to charge the group 1. Then DC-DC1 is stopped and DC-DC3 is driven by PWM3 to discharge the group 1 into the group 2 with a very short time. And then DC-DC1 is driven again by PWM1 and DC-DC3 is stopped. Therefore, the work sequence of group 1 is: charging, stop charging, discharging, stop discharging, and charging again. Similarly, when group 2 achieved the threshold value, the same working sequence is implemented. By this means, the two groups of batteries discharge to each other with a very fast period to improve their acceptable capacity of charging current. The discharging time is far shorter than charging time. When the two groups of batteries both achieved the threshold value of fast charging model, the modules of DC-DC1 and DC-DC2 are working alternatively. During charging period of any one group, another group stopped charging and will discharge to the charging group by DC-DC3 or DC-DC4 with a very short time. In this situation, DC-DC4 will only be driven during DC-DC1 working period, which means that DC-DC4 and DC-DC2 must not work at the same time. Similarly, DC-DC3 will only be driven during DC-DC2 working period, which means that DC-DC3 and DC-DC1 must not work at one time. Each DC-DC module is single direction conducting and driven by PWM signals generated by MPPT controller.

In terms of literatures [9] and [10], the MPPT controller with design shown as Fig.3 can provide fast charging performance. For the discharging load is batteries, there is no extra energy to waste. Using the design proposed in this paper in a PV system, the utilization efficiency of solar energy can be improved comprehensively. 


\section{Conclusion}

The design of independent PV system should consider not only the MPPT function and the protection for batteries, but also the charging speed. To provide fasting charging performance, this paper proposed a new controller topology which combined MPPT algorithm and fasting charging model. By this means, the redundancy of batteries capacity for keeping work during continual rainy days can be charged fully at one time, which can avoid that the batteries work in undervoltage condition. Thus the service period of batteries can be prolonged and the utilization efficiency of energy can be improved comprehensively.

\section{Acknowledgement}

In this paper, the research was sponsored by the Nature Science Foundation of China (Project No. 61563023), Youth Science Foundation of Jiangxi Province (Projects No. 20132BAB211038, 20151BAB207013), Nature Science Foundation of Jiangxi Province (Project No. 20142BAB207006), and Science and Technology Foundation of Jiangxi Province Department of Education (Project No. GJJ14743).

\section{References}

[1] Xiao W, Ozog N, Dunford WG. Topology study of photovoltaic interface for maximum power point tracking [J]. IEEE Trans Ind Electron, 2007. 54(3): 1696-704.

[2] Hsiao YT, Chen CH. Maximum power point tracking for photovoltaic power system [C]. In: $37^{\text {th }}$ IAS annual meeting; 2002. 1035-40.

[3] Hussain KH, MutaI. Maximum photovoltaic power tracking: analgorithm for rapidly changing atmospheric conditions [C]. In: Proceeding of industrial electrical engineering, generation transmission and distribution. 1995.142: 59-64.

[4] Patcharaprakiti N, Premrudeepreechacharn S. Maximum power point tracking using adaptive fuzzy logic control for grid connected photovoltaic system [J]. IEEE Power Eng. 2002. 372-7.

[5] Parimita M, Bhuvaneswari G, Balasubramanian R, Navdeep KD. MATLAB based modeling to study the performance of different MPPT techniques used for solar PV system under various operating conditions [J]. Renewable and Sustainable Energy Reviews. 2014. 38: 581-593

[6] Yang H, Wang H, Wang XD, Xiao Y, Cui RQ, Zhou ZHB. Study on overcharging protection and temperature compensation of VRLA battery renewable energy system [J]. Acta Energiae Solaris Sinica, 2001, 22(2): 223- 225.

[7] Xiao JM, Du YH, Li YB, Zhu HM, Liu PC. Study on two- stage fast charge method for VRLA batteries in PV systems [J]. Chinese Labat Man. 2012. 1(49): 41-48.

[8] Wang CK, Xie LL, Luo MJ. Design and simulation for small wind and solar control systems based on three-stage battery charging [J]. Computer measurement \& control. 2012. 20(7): 1867-1869.

[9] Joseph A Mass. Wide Temperature Range Charging System Controlled by Battery Acceptance [M]. Society of Automotive Engineers, 1968.

[10] Guo YF. Design and Analysis of Charging System with Negative Pulse of Lead-acid Battery [J]. Low Voltage Apparatus, 2011, (8):23-25 